\title{
Tightening a Bond with a Voltage
}

\section{A scheme that uses a voltage to control a chemical bond's strength allows the tip of an atomic force microscope to pluck a graphene sheet from a substrate.}

By Matteo Rini

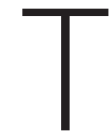

oday, myriad techniques exist for using atoms as microscopic "Legos" that can be assembled into complex nanostructures. Now, a team led by Jörg Kröger of the Technical University of Ilmenau, Germany, has expanded the nanomanipulation toolkit with a technique for controlling an individual chemical bond's strength [1]. Applying a voltage across the bond, they showed that they could weaken or strengthen the interaction between an atom at the tip of an atomic force microscope and an atom in a graphene sheet. The scheme could lead to methods for applying mechanical loads in nanomachines or for studying how bond strength affects chemical reactions.

The new technique exploits the behavior of polar covalent bonds, which form between two atoms with different electron affinity, such as the hydrogen and oxygen atoms in a water molecule. In such molecules, the bonding electrons are unequally distributed between the two atoms, so an applied

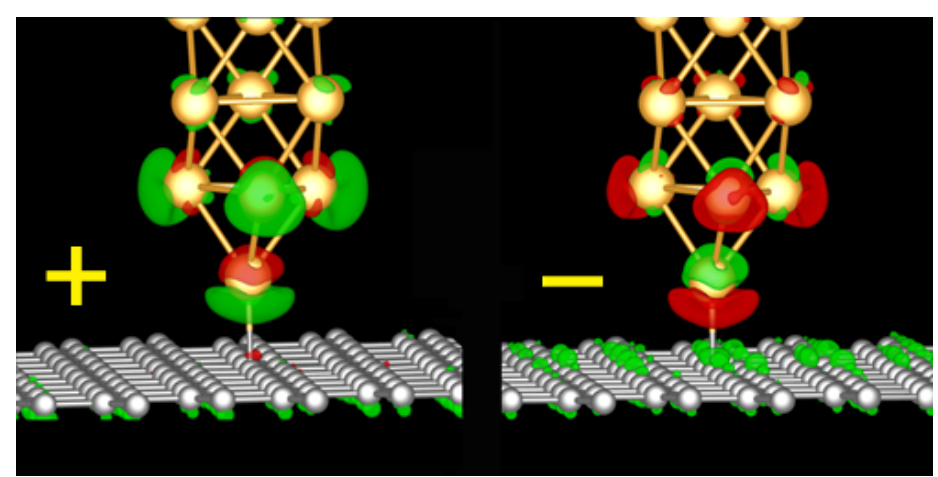

Credit: M. Omidian et al. [1] electric field can change the electron distribution, which affects the bond strength.

In their setup, the team placed the tip of an atomic force microscope probe on top of a graphene sheet. The tip was decorated with a gold atom, which formed a bond with one of the graphene sheet's carbon atoms. The team then applied a small voltage between the graphene sheet and the tip.

The team found that negative voltages weakened the bond, while positive voltages strengthened it. For the largest positive voltages, this strengthening was so large that when the tip got retracted, the gold atom stuck to the carbon atom and the tip acted like a crane that pulled up the whole graphene sheet. The team's simulations showed that the effect comes from a voltage-induced charge redistribution in the atoms' atomic orbitals.

Matteo Rini is the Editor of Physics.

\section{REFERENCES}

1. M. Omidian et al., "Electric-field control of a single-atom polar bond," Phys. Rev. Lett. 126, 216801 (2021). 